\title{
BMJ Open Screening for the metabolic side effects of antipsychotic medication: findings of a 6-year quality improvement programme in the UK
}

\author{
T R E Barnes, ${ }^{1,2}$ S F Bhatti, ${ }^{2}$ R Adroer, ${ }^{2}$ C Paton ${ }^{1,2}$
}

To cite: Barnes TRE, Bhatti SF, Adroer R, et al. Screening for the metabolic side effects of antipsychotic medication: findings of a 6 year quality improvement programme in the UK. BMJ Open 2015;5:e007633. doi:10.1136/bmjopen-2015007633

- Prepublication history for this paper is available online. To view these files please visit the journal online (http://dx.doi.org/10.1136/ bmjopen-2015-007633).

Received 26 January 2015 Revised 21 July 2015 Accepted 4 August 2015

\section{CrossMark}

${ }^{1}$ The Centre for Mental Health, Imperial College London, Hammersmith Hospital Campus, London, UK

${ }^{2}$ Prescribing Observatory for Mental Health (POMH-UK), Royal College of Psychiatrists, London, UK

Correspondence to Professor TRE Barnes; t.r.barnes@imperial.ac.uk

\section{ABSTRACT}

Objectives: To increase the frequency and quality of screening for the metabolic syndrome in people prescribed continuing antipsychotic medication.

Design: An audit-based, quality improvement programme (QIP) with customised feedback to participating mental health services after each audit, including benchmarked data on their relative and absolute performance against an evidence-based practice standard and the provision of bespoke change interventions.

Setting: Adult, assertive outreach, community psychiatric services in the UK.

Participants: 6 audits were conducted between 2006 and 2012. 21 mental health Trusts participated in the baseline audit in 2006, submitting data on screening for 1966 patients, while 32 Trusts participated in the 2012 audit, submitting data on 1591 patients.

Results: Over the 6 years of the programme, there was a statistically significant increase in the proportion of patients for whom measures for all 4 aspects of the metabolic syndrome had been documented in the clinical records in the previous year, from just over 1 in 10 patients in 2006 to just over 1 in 3 by 2012. The proportion of patients with no evidence of any screening fell from almost $1 / 2$ to 1 in 7 patients over the same period.

Conclusions: The findings suggest that audit-based QIPs can help improve clinical practice in relation to physical healthcare screening. Nevertheless, they also reveal that only a minority of community psychiatric patients prescribed antipsychotic medication is screened for the metabolic syndrome in accordance with best practice recommendations, and therefore potentially remediable causes of poor physical health remain undetected and untreated.

\section{INTRODUCTION}

People with schizophrenia have an excess mortality, 2 to 3 times higher than the general population; life expectancy is shortened by 15-20 years. ${ }^{1}$ This differential mortality gap appears to have increased over recent
Strengths and limitations of this study

- The findings regarding screening are based on self-report data from secondary care mental health services, and relate only to measures and assessments that were documented in the clinical records.

- The generalisability of these findings rests on the large national sample sizes; at each audit, each participating clinical team was asked to submit screening data for all eligible patients on their caseload, so systematic bias in the selection of cases is unlikely.

- Information was not systematically collected from participating mental health services on the generation and implementation of local action plans to tackle areas where practice fell short of the standard or the use of the bespoke change interventions.

- The extent to which the improvement seen in physical healthcare monitoring in patients prescribed continuing antipsychotic medication can be attributed to the participation of services in the quality improvement programme is uncertain as there were other national initiatives raising awareness of the need for such monitoring over the 6-year period.

decades. ${ }^{23}$ Approximately $60 \%$ of this excess mortality is due to physical illness. ${ }^{3}$ This may be partly because, for various reasons, physical illnesses in people with schizophrenia may be diagnosed late and not optimally treated. Other reasons are associated with the psychotic illness itself as well as illness-related factors such as physical inactivity, cigarette smoking, excess alcohol consumption and poor diet. However, treatment with antipsychotic medication is also a contributory factor, not least because of the metabolic side effects, including weight gain. ${ }^{34}$

Hypertension, central obesity, raised fasting glucose and dyslipidaemia, when clustered together, are highly predictive of 
cardiovascular disease and type 2 diabetes. Individuals who have at least three of these risk factors have been described as having metabolic syndrome (MS). MS is relatively common in people on long-term antipsychotic treatment, with a prevalence varying from $6 \%$ to over $60 \% .^{5-8}$ All four aspects of the MS are potentially remediable. Antipsychotic-induced weight gain and the metabolic side effects can be difficult to manage, but there is a range of possible interventions. These include advice on lifestyle, diet and exercise, ${ }^{9}$ switching to an antipsychotic with a lower liability for such adverse effects, ${ }^{10}$ augmentation of antipsychotic medication with drugs such as metformin or topiramate, and augmentation of clozapine and possibly olanzapine with aripiprazole. ${ }^{11-13}$

There is a consensus across evidence-based guidelines that patients on continuing antipsychotic medication should receive regular metabolic monitoring and adequate treatment of any cardiometabolic risk factors identified. In routine clinical practice, such monitoring tends to fall well short of relevant guideline recommendations. ${ }^{14} 15$ This paper reports on an audit-based, quality improvement programme (QIP) in the UK targeted at screening for the MS in community psychiatric patients on continuing antipsychotic medication.

\section{METHODS}

The Prescribing Observatory for Mental Health $(\mathrm{POMH}-\mathrm{UK})^{16}$ invited all National Health Service (NHS) Trusts and other private or charitable healthcare organisations (hereafter referred to as Trusts) in the UK providing specialist mental health services to participate in an audit-based QIP focusing on screening for the MS in people prescribed antipsychotic medication under the care of assertive outreach community psychiatric services. All services were self-selected in that they chose to participate, and each participating clinical team was asked to review the clinical records of every patient under their care who was prescribed antipsychotic medication at the time of the audit.

The practice standard for audit was that all patients prescribed continuing antipsychotic medication should have their blood pressure, body mass index (BMI; or other measure of obesity), blood glucose (or glycated hemoglobin (HbAlc)) and lipids measured at least once a year. Annual screening was considered the minimum acceptable practice; most relevant national guidelines recommend more frequent screening of some or all of these measures depending on the drug prescribed or a patient's demographic or clinical characteristics. The audit tool was developed by the POMH-UK team, advised by an expert steering group and staff from mental health NHS Trusts attending POMH-UK regional workshops. Clinical audits were conducted in 2006 (baseline) and 2007 (reaudit), and subsequently, supplementary audits were carried out in 2008, 2009, 2010 and 2012. At each audit, the data collected on each patient included the following: (1) demographic data: age, gender and ethnicity; (2) clinical data: International Classification of Diseases 10th Revision (ICD-10) mental health diagnoses, known diagnosis of diabetes, hypertension or disturbed plasma lipid profile; (3) medication information: details of the antipsychotic medication currently prescribed including whether it was a firstgeneration (FGA) or second-generation (SGA) antipsychotic drug and whether it constituted a high-dose prescription (high dose was defined as an antipsychotic drug prescribed at a daily dose above the maximum recommended in the British National Formulary $(\mathrm{BNF})^{17}$ or, if a patient were prescribed more than one antipsychotic, where the cumulative percentage of the BNF maximum dose for the drugs was more than $100 \%$ ), other medication prescribed; and (4) monitoring data: whether there was documentation in the clinical records that there had been assessment of the four aspects of the MS-blood pressure, obesity status (BMI or weight and height or waist circumference or waist-hip ratio), blood glucose (random or fasting blood glucose, HbAlc or glucose tolerance test) and plasma lipid profile (high-density lipoprotein and low-density lipoprotein and/or total cholesterol) in the previous year, as well as whether the documented tests had been carried out by a mental health team, an acute general hospital team or in primary care.

After each audit, customised reports were sent to each participating Trust. The first section of the report summarised the national data while the second provided each Trust with their own performance data, benchmarked anonymously against the performance of the other participating Trusts, thus allowing review of both relative and absolute performance against the standard for screening of the four MS aspects. The final section of the report allowed for comparison of performance across the individual clinical teams within a Trust.

\section{Change intervention}

In 2006, a questionnaire was sent out to all participating Trusts to gather information on potential barriers to screening as well as possible facilitating factors. The findings of the questionnaire have been reported elsewhere $^{14}$ and, along with the analysis of the data collected in the baseline audit, informed the development of change interventions. The main one was a poster indicating the normal ranges for test results for the four aspects of the MS, the range of borderline high results that would warrant lifestyle advice and/or additional monitoring, and the threshold levels of elevated results that should prompt referral for review by a general practitioner or medical team. This poster was made available to all participating Trusts to support their local action plans, prompted by the findings in their individual audit reports. In addition, a lifestyle management pack was developed, which provided guidance and resources for staff and service users relating to aspects of physical health such as diet, physical exercise and 
smoking cessation. There was also an intervention designed for service users: a physical health check reminder card.

\section{Statistical analysis}

Analysis of the data was performed using the computer software, SPSS (IBM SPSS statistics V.21, Chicago, Illinois, USA). Simple descriptive statistics were used to examine the demographic and clinical characteristics of the patient sample and describe prescribing practice, including performance against the clinical practice standard.

To evaluate the trend in outcome over the duration of the programme, the data analysis used three outcome variables, all of which are related to the practice standard for the clinical audits. These three variables, measured at the individual patient level, were: no evidence of MS screening in the past year; some evidence of MS screening in the past year and documentation in the past year of a test result for all four aspects of MS. A feature of the data was that different combinations of mental health Trusts took part at each clinical audit, and there was variability in outcomes between Trusts. Therefore, the analysis needed to take account of variations in participating Trusts over time. Given the data structure and the binary nature of the outcomes, a multilevel logistic regression was performed. Two-level models were used with individual patients nested within Trusts. To allow for an easier interpretation of the results, a consistent trend over time was assumed by using a linear term for year of audit.

Further, a binary logistic regression was performed on data from the 2012 sample to examine the clinical factors associated with whether or not patients had evidence of all four measurements of physical health, namely documented results for blood pressure, obesity status, blood glucose and lipid profile, in the past year, or three or fewer measures. A logistic regression was performed in two stages. First, the separate association between each variable and the outcome was examined in a series of univariable analyses. The second stage jointly examined associations with the outcome in a multivariable analysis. To simplify the final regression model, a backwards selection procedure was performed to retain only the statistically significant variables. This involves omitting non-significant variables, one at a time, until only the significant variables remain.

\section{RESULTS}

For each audit between 2006 and 2012, the number of participating Trusts and the size of the total national sample of patients, cared for within assertive outreach teams, are shown in table 1 . The demographic and clinical characteristics of the 1591 community patients in the 2012 national sample are shown in table 2 .

Table 1 shows the proportion of the sample for whom there was no documented evidence in their clinical records of screening for any aspect of the MS in the previous year, the proportion who had documented evidence of partial screening (mention of review of any of the four aspects of the MS and/or documentation of up to three relevant test results) and those who had documented test results for all four aspects. High-dose antipsychotic medication was prescribed for $8 \%$ of the national sample in 2012, while the respective figure for combined antipsychotics was $19 \%$.

Figure 1 shows the proportion of patients in each of these clinical audits for whom test results for obesity, blood pressure, plasma glucose and plasma lipid profile were documented in the clinical records during the previous year. A higher percentage indicates that practice was closer to the audit standard for that aspect of the MS during the past year. In 2006, the proportions of patients in the national sample with a result or measure in their clinical records for blood pressure, obesity/BMI, blood pressure, plasma glucose and lipid profile were $17 \%, 26 \%, 28 \%$ and $22 \%$, respectively. By 2012 , the corresponding figures were $58 \%, 59 \%, 52 \%$ and $50 \%$. The data in figure 2 reveal that, in the 2012 supplementary audit, the majority of the assessments documented in the clinical records in mental health services had been undertaken by mental health teams rather than by primary care.

Table 1 shows the proportion of the patients in the national samples from 2006 to 2012 who were screened for all four aspects of the MS. At each audit, from 2006 to 2012 , there was marked variation across the participating Trusts. In 2012, the proportion of patients in Trust samples with a test result for all four MS aspects varied

Table 1 Level of documented screening for the metabolic syndrome (MS) in the total national sample at each audit from 2006 to 2012

\begin{tabular}{|c|c|c|c|c|c|c|}
\hline & \multicolumn{6}{|c|}{ Clinical audit } \\
\hline & 2006 & 2007 & 2008 & 2009 & 2010 & 2012 \\
\hline Number of participating Trusts & 21 & 21 & 13 & 21 & 29 & 32 \\
\hline Number of participating clinical teams & 48 & 35 & 28 & 56 & 76 & 100 \\
\hline Number of patients in sample & 1966 & 1516 & 1035 & 2522 & 3058 & 1591 \\
\hline No evidence of MS screening & $46 \%$ & $25 \%$ & $31 \%$ & $28 \%$ & $27 \%$ & $14 \%$ \\
\hline Some evidence of MS screening & $43 \%$ & $52 \%$ & $50 \%$ & $50 \%$ & $49 \%$ & $52 \%$ \\
\hline Test result documented for all four aspects of MS & $11 \%$ & $23 \%$ & $18 \%$ & $22 \%$ & $24 \%$ & $34 \%$ \\
\hline
\end{tabular}


Table 2 Demographic and clinical characteristics of the 2012 national audit sample

\begin{tabular}{ll}
\hline & Total national \\
& sample \\
& N=1591 \\
\hline Gender: \% male/\% female & $67 / 33$ \\
Age (years): mean (SD) & $43(11)$ \\
Ethnicity (\%) & \\
White/White British & 69 \\
Black/Black British & 12 \\
Asian/Asian British & 9 \\
Mixed/other & 5 \\
Not collected & 4 \\
Not stated/declined & $<1$ \\
Psychiatric diagnosis, ICD-10 code (\%) & \\
F00-F09: organic, including & 2 \\
symptomatic, mental disorders & \\
F10-F19: mental and behavioural & 1 \\
disorders due to psychoactive & \\
substance use & \\
F20-29: schizophrenia, schizotypal and & 72 \\
delusional disorders & \\
F30-39: mood (affective) disorders & 13 \\
F40-48: neurotic, stress-related and & 1 \\
somatoform disorders & \\
F50-59: behavioural syndromes & $<1$ \\
associated with physiological & \\
disturbances and physical factors & \\
F60-69: disorders of adult personality & 6 \\
and behaviour & \\
F70-79: mental retardation & \\
F80-89: disorders of psychological \\
development & \\
F90-98: behavioural and emotional \\
disorders with onset usually occurring \\
in childhood and adolescence & \\
F99: unspecified mental disorder & \\
Not known & \\
\hline ICD-10, International Classification of Diseases & 1 \\
\hline & \\
\hline
\end{tabular}

from $0 \%$ to $70 \%$. The proportion of the national sample with no evidence of screening of any aspect of the MS over the previous year fell during the course of the QIP, from $46 \%$ in 2006 to $14 \%$ in 2012.

A multilevel logistic regression analysis was conducted to examine the trend in outcome of MS screening performance over the 6 years. The results are reported in table 3 in the form of ORs, indicating the relative change in the odds of an outcome occurring for a 1-year increase in time. Corresponding CIs for the ORs are also reported, along with $\mathrm{p}$ values indicating the significance of the results. The results show that the odds of 'no evidence' of screening occurring decreased by around a quarter for each year of the programme. Over the same period, there was an increase in the proportion of patients with some evidence of MS screening and of those for whom all four aspects of the MS had been documented in the previous year. The increase in the proportion of patients with some evidence of screening was smaller: the odds of this level of screening occurring increased by $6 \%$ a year. There was a larger increase in the proportion with documentation of all four aspects of MS, with the odds of this outcome occurring increasing by just over a quarter each year.

To explore which clinical factors might be related to the likelihood of full screening, we examined the clinical characteristics of those patients in the 2012 audit sample with documented results for all four MS variables within the past year. A series of univariable logistic regression analyses were conducted to examine the separate association between each variable and having all four MS measurements documented in a patient's clinical records within the past year. The variables tested were age, gender, ethnicity, a known diagnosis of diabetes, a known diagnosis of hypertension, a known diagnosis of dyslipidaemia, prescription of a depot/long-acting antipsychotic preparation as the only antipsychotic medication, prescription of a combination of depot/long-acting antipsychotic preparation and oral antipsychotic, prescription of an FGA oral antipsychotic, prescription of a FGA depot antipsychotic, prescription of clozapine, prescription of a SGA oral antipsychotic other than clozapine, prescription of more than one antipsychotic drug and prescription of high-dose antipsychotic medication. Those variables with a significant association with having all MS measurements documented were examined in a multivariable analysis. A backwards selection procedure was used to retain only the statistically significant variables, and the final model resulting from this process is summarised in table 4 .

The multivariable results suggested that being prescribed a depot/long-acting antipsychotic preparation as the only antipsychotic medication, a known diagnosis of diabetes and a known diagnosis of dyslipidaemia were all independently associated with having all MS measurements documented. As in the univariable analyses, patients with diabetes and dyslipidaemia were more likely to have all four measurements in their clinical records, while patients receiving only a depot antipsychotic were less likely to have all four measurements documented. After adjusting for the effects of these three variables, there were no additional effects of ethnicity, FGA oral medication or known diagnosis of hypertension, all of which were statistically significant in the univariable analyses.

\section{DISCUSSION}

The National Institute for Health and Care Excellence (NICE) psychosis and schizophrenia guideline published in $2014^{18}$ specifically recommended that appropriate physical health checks, including the MS variables addressed in this QIP, should take place annually, which is in line with the practice standard we had audited against since 2006. Over the 6 years of the POMH-UK QIP, the audits against this standard, in samples of people prescribed antipsychotic medication under the 
Figure 1 Proportion of patients in the total national sample with documented metabolic syndrome measures in their clinical records: 2006-2012 (BMI, body mass index).
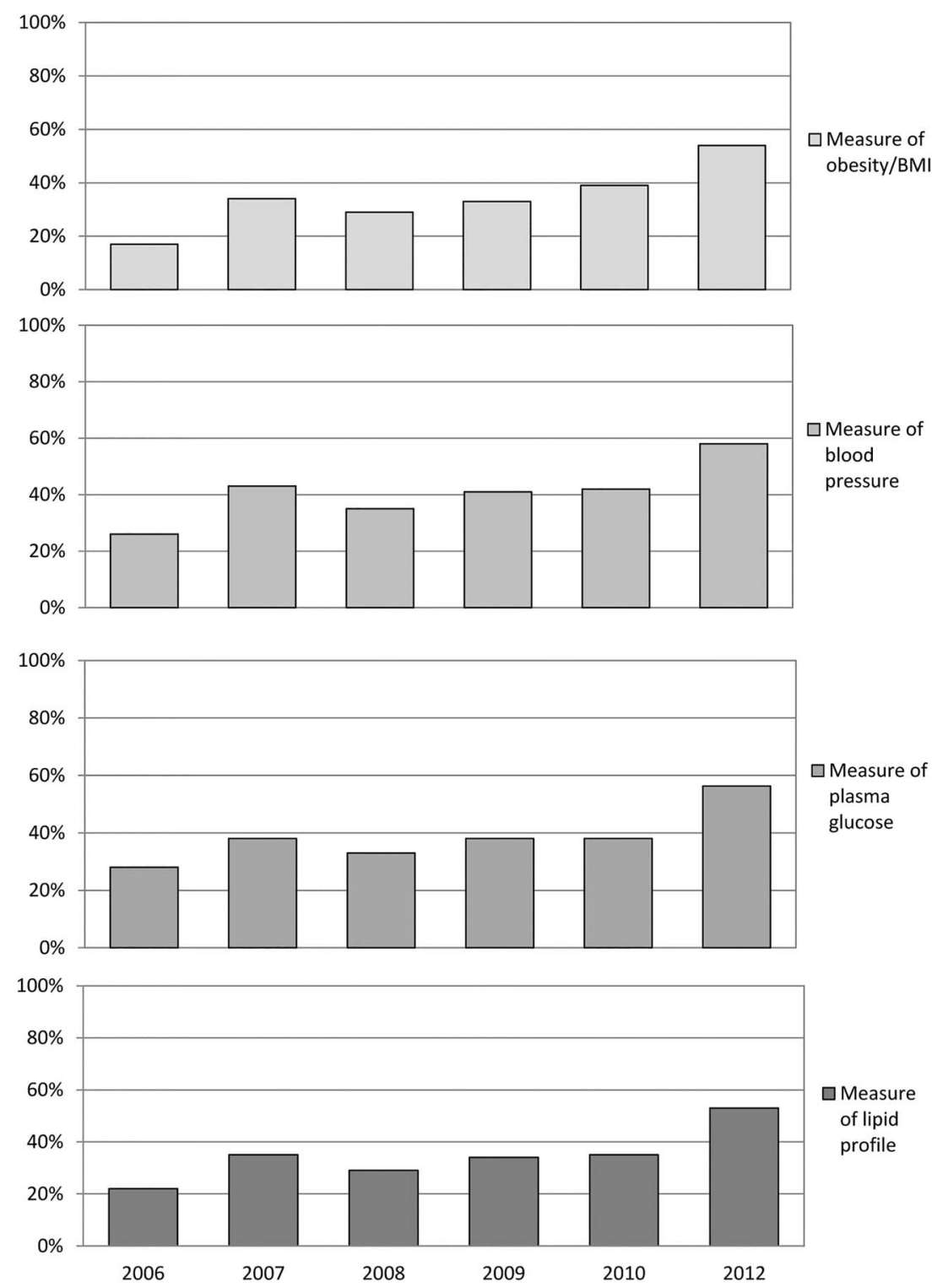

care of assertive outreach services, revealed an improvement in the frequency and breadth of screening for the MS. The successive national audit samples show an increase in the proportion of patients for whom there were documented measures for all four aspects of the MS in the previous year, from just over 1 in 10 patients

Figure 2 Clinical service/team that conducted metabolic syndrome tests in the 2012 audit (BMI, body mass index; GP, general practitioner).

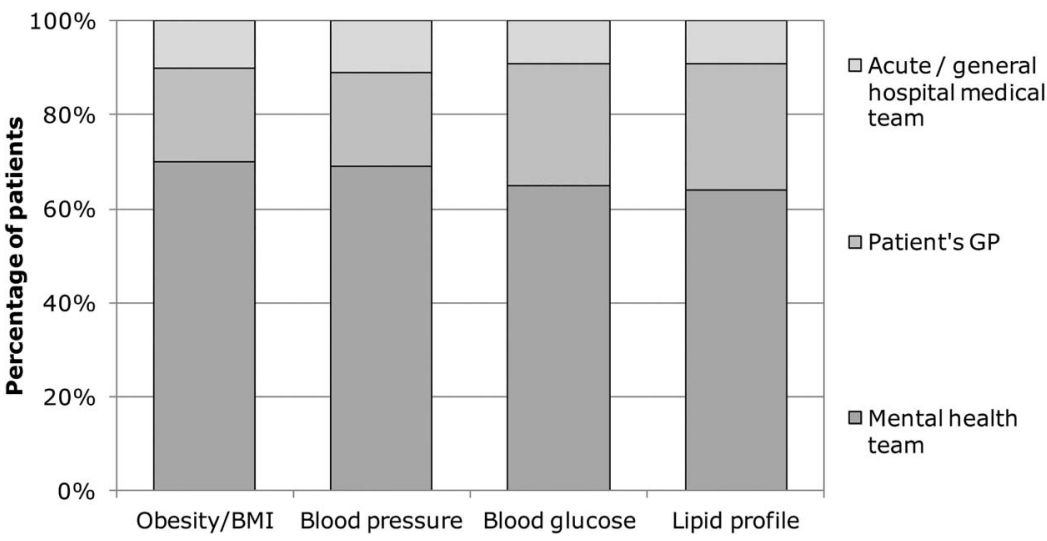


Table 3 Trend in the outcome of metabolic syndrome (MS) screening performance over the 6 years of the quality improvement programme: results of a multilevel logistic regression analysis

\begin{tabular}{lll}
\hline Outcome & OR $(95 \% \mathrm{Cl})^{*}$ & $\begin{array}{l}\mathbf{p} \\
\text { Value }\end{array}$ \\
\hline $\begin{array}{l}\text { No evidence of MS screening } \\
\text { Some evidence of MS }\end{array}$ & $0.73(0.70$ to 0.75$)$ & $<0.001$ \\
$\begin{array}{l}\text { screening } \\
\text { Result documented for all }\end{array}$ & $1.27(1.03$ to 1.09$)$ & $<0.001$ \\
four aspects of MS & & \\
\hline *OR represents the relative change in the odds of the outcome for \\
the increase in time between audits.
\end{tabular}

in 2006 to just over 1 in 3 in 2012. An initial increase in screening from the baseline audit in 2006 to reaudit in 2007 , with a doubling of the proportion of patients with evidence of full screening, with documentation of all four aspects of the MS, was maintained over the subsequent audits, further improvement not being evident until the 2012 supplementary audit. By 2012, only 14\% of the national sample had no evidence of any screening, compared with $46 \%$ in 2006.

The generalisability of these findings rests on the large national sample sizes. For the majority of the audits, a third or more of all the mental Trusts in England participated, and in 2012, it was well over half. For each audit, participating clinical services were asked to submit screening data on all eligible patients on their caseload, so systematic bias in the selection of cases is unlikely. However, when interpreting the data collected, a further issue is whether any change in the level of screening seen over time might be partly attributable to variation in the proportion of the total patient sample contributed by each of the Trusts at each audit. When addressing the changes in screening practice seen between the 2006 baseline audit and the 2007 reaudit, we performed a multilevel logistic regression to correct for such differences between Trusts. The results indicated that, for all four MS measures, there was a statistically significant difference between baseline and reaudit, with no apparent bias introduced into the findings by the variation in the number of Trusts participating on the two occasions. ${ }^{19}$ The results of a multilevel logistic regression analysis of the trend in MS screening data across all six audits, which also took account of variations in participating Trusts over time, provided evidence of a statistically significant fall over the course of the programme in the proportion of patients who had no evidence of MS screening over time and a significant increase in the proportion of patients with partial or full MS screening.

As in all the audits conducted as part of this QIP, variation in current screening practice across the participating Trusts in the 2012 supplementary audit was marked. For those Trusts that had taken part in the previous audits, the level of screening for the MS had also varied over time. During the QIP, we had suggested to Trusts that when they developed local action plans to address any shortfall between their prescribing performance and the practice standard, they might wish to take account of possible influences, such as the degree to which local strategies to ensure screening had already been implemented, whether they had selected different patient samples or even different assertive outreach services to participate at different stages, and whether there had been any relevant changes in staffing, caseload or service delivery. We did not systematically collect information from the participating mental health services regarding reflection on their practice in the light of the benchmarked performance data provided, whether they had generated and implemented local action plans to tackle areas where practice fell short of the standard or whether they had used any of the change interventions provided. However, there is evidence from published audit reports and Trust quality accounts that review of the POMH audit data stimulated such activity. ${ }^{20-26}$

The extent to which the improvement seen in the proportion of patients prescribed continuing antipsychotic medication who had physical healthcare screening over the past year can be attributed to the QIP is uncertain as there were other national initiatives raising awareness of the need for such screening over the 6 -year period. The NICE schizophrenia guidelines in 2002 and $2009^{27} 28$ recommended such monitoring, as did the Maudsley Prescribing Guidelines. ${ }^{29}$ In addition, NICE issued guidelines on cardiovascular risk assessment focusing on modification of blood lipids in people at high risk ${ }^{30}$ in 2008, and the Royal College of Psychiatrists published a report on physical health and mental health ${ }^{31}$ in 2009. In 2012, some locally developed schemes within the

Table 4 Multivariable analysis of effect of potential explanatory variables on documentation of measures for all four aspects of the metabolic syndrome in the past year $(n=1591)$

\begin{tabular}{|c|c|c|c|}
\hline Variable & Category & OR $(95 \% \mathrm{Cl})$ & p Value \\
\hline $\begin{array}{l}\text { Prescribed depot/long-acting antipsychotic preparation as the only } \\
\text { antipsychotic medication }\end{array}$ & $\begin{array}{l}\text { No } \\
\text { Yes }\end{array}$ & $10.62(0.49$ to 0.78$)$ & $<0.001$ \\
\hline Known diagnosis of diabetes & $\begin{array}{l}\text { No } \\
\text { Yes }\end{array}$ & $11.52(1.10$ to 2.10$)$ & 0.01 \\
\hline Known diagnosis of dyslipidaemia & $\begin{array}{l}\text { No } \\
\text { Yes }\end{array}$ & 12.13 (1.62 to 2.80$)$ & $<0.001$ \\
\hline
\end{tabular}


Commissioning for Quality and Innovation (CQUIN) framework, a 'pay-for-performance' scheme, included screening for the metabolic side effects of antipsychotic medication as a target: whether such schemes improve health outcomes remains unclear. ${ }^{32}$ Further, since 2004, general practitioners in England and Wales have been incentivised within the Quality and Outcomes Framework $(\mathrm{QOF})^{33}$ to offer such physical healthcare monitoring annually for people with severe mental illness, although this finished in 2014. In our QIP audit in 2012, the MS screening tests documented in the clinical records had been undertaken by the mental health teams in over two-thirds of cases, although results from primary care were available in the mental health clinical records in a small proportion of patients. The extent to which these tests were duplicated in primary care under the QOF but not communicated to mental health teams is unknown.

Despite these guidelines and initiatives and the improvements seen over the period of the POMH-UK QIP, the extent and frequency of screening reported by 2012 was still well below best practice recommendations. Some of the barriers to screening in practice were identified in the early stages of the $\mathrm{QIP}^{14}$ and included uncertainty as to whether such physical health screening was the responsibility of the psychiatric team rather than a primary care clinician, a lack of confidence in the interpretation of abnormal screening results and limited access to basic equipment such as a tape measure and weighing scales. Another possible explanation for the relatively low level of screening is that clinicians may target patients for the assessment of metabolic side effects rather than routinely screen all patients prescribed continuing antipsychotic medication. Analysis of the reaudit data in 2008 revealed that those psychiatric patients with a known diagnosis of dyslipidaemia or diabetes, as well as those prescribed clozapine, were being targeted for screening in that they were more likely to have had all four MS measures documented in the past year. As previously noted, ${ }^{19}$ this association between screening and clozapine treatment may reflect clinicians' perception of the risk of metabolic side effects with this drug as well as the opportunity for blood samples afforded by the monitoring systems in place as part of the prescription of the drug. The association with diagnoses of diabetes and dyslipidaemia suggests that such diagnoses prompt more frequent screening for metabolic problems and/or that diabetes and dyslipidaemia are more likely to be detected with more frequent screening. When the data from the 2012 sample were analysed, known diagnoses of dyslipidaemia or diabetes emerged again as significant predictors of full screening, while a patient prescribed a depot/long-acting antipsychotic preparation as their only antipsychotic treatment was less likely to have all four MS measures documented. Receiving only a depot antipsychotic may be a proxy for variables which were not collected in the audit, such as level of engagement with clinical services or frequency of medication review, or possibly the perception by clinicians that at least some of the antipsychotic drugs commonly prescribed as depot/ long-acting preparations have a lower liability for metabolic side effects.

The POMH-UK audit-based programmes demonstrate a workable and effective methodology for quality improvement in the NHS and also provide Trusts with evidence of implementation of national treatment guidelines into practice. Any success this particular programme achieved may be partly attributed to the use of a widely accepted practice standard and educational and facilitative change interventions designed to directly address barriers to physical healthcare screening that had been originally identified by participating clinical teams. ${ }^{14} 19$ The improvement in the frequency and quality of screening for the metabolic side effects of antipsychotics seen over the 6 years of this POMH-UK programme should provide more opportunities for timely, evidence-based management when such effects are identified in individual patients. This, in turn, has the potential to enhance patient safety and long-term health outcomes.

Acknowledgements Acknowledgements are due to the POMH-UK leads in all participating Trusts and healthcare organisations and the clinicians and administrators who collected and submitted the audit data. Thanks are also due to Paul Bassett for statistical analyses as well as the members of the POMH-UK team aside from the co-authors of this paper: Susan Lemmey, Krysia Zalewska, Sonya Chee and Oda Skagseth.

Contributors All four authors contributed to the conception or design of the data collection tool, and the acquisition, analysis or interpretation of data for the work. The paper was written by TREB and CP but SFB and RA revised it critically for important intellectual content. All four authors approved the final version submitted for publication and agreed to be accountable for the accuracy or integrity of any part of the work.

Funding POMH-UK was originally funded by a tapering grant from an independent charity, the Health Foundation, under its 'Engaging with Quality' initiative. It is now funded entirely from the subscriptions of member mental health services, principally mental health NHS Trusts.

Competing interests In the past 3 years, TREB has received honoraria from Roche for speaking at educational meetings and been a member of scientific advisory boards for Sunovion and Otsuka/Lundbeck and CP has undertaken consultancy work for Sunovion.

Provenance and peer review Not commissioned; externally peer reviewed.

Data sharing statement The following statement on data ownership was included in the customised reports to all participating Trusts for this quality improvement programme: In line with the original memorandum of understanding between $\mathrm{POMH}-\mathrm{UK}$ and member healthcare organisations (predominantly mental health NHS Trusts), the following statement outlines the agreement regarding ownership of the audit data in this quality improvement programme. Ownership of the local data submitted to POMH-UK is retained by the healthcare organisation that submitted them. These data have been made available to POMH-UK in a way that is anonymous, with the exception of the identity of the source organisation. The aggregate data from all participating organisations have been analysed by POMH-UK, to produce this customised report. This report summarises the national results and local results at organisation and clinical team level, benchmarked anonymously against the other organisations taking part. There is a publication strategy allowing POMH-UK to publish the anonymous aggregated data on its website and/or in appropriate scientific journals. Any requests from other organisations for these audit data will be referred to the POMH-UK reports 
appearing in the public domain or provided with a list of member healthcare organisations and asked to approach then individually. It is each organisation's decision whether, and with whom, to share their data. Reflection by clinical teams on their benchmarked performance is perhaps the most potent element of POMH-UK programmes. In addition to performance against the clinical standards, the audit data include demographic, diagnostic and other relevant clinical information that provide a context for interpretation and understanding of practice, which can inform local strategies and systems to achieve improvement. The data collected are designed to be suitable for this clinical purpose, and not for objective ranking of healthcare organisations, for which they are untested and would not necessarily be appropriate.

Open Access This is an Open Access article distributed in accordance with the Creative Commons Attribution Non Commercial (CC BY-NC 4.0) license, which permits others to distribute, remix, adapt, build upon this work noncommercially, and license their derivative works on different terms, provided the original work is properly cited and the use is non-commercial. See: http:// creativecommons.org/licenses/by-nc/4.0/

\section{REFERENCES}

1. Laursen TM, Nordentoft M, Mortensen PB. Excess early mortality in schizophrenia. Annu Rev Clin Psychol 2014;10:425-48.

2. Saha S, Chant D, McGrath J. A systematic review of mortality in schizophrenia: is the differential mortality gap worsening over time? Arch Gen Psychiatry 2007;64:1123-31.

3. Laursen TM, Munk-Olsen T, Vestergaard M. Life expectancy and cardiovascular mortality in persons with schizophrenia. Curr Opin Psychiatry 2012;25:83-8.

4. Berrocal-Izquierdo N, Bernardo M. Schizophrenia and cerebrovascular disease. A description of a series and bibliographic review. Actas Esp Psiquiatr 2014;42:74-82.

5. Vancampfort D, Wampers M, Mitchell AJ, et al. A meta-analysis of cardio-metabolic abnormalities in drug naïve, first-episode and multi-episode patients with schizophrenia versus general population controls. World Psychiatry 2013;12:240-50.

6. Malhotra N, Kulhara $\mathrm{P}$, Chakrabarti S, et al. A prospective, longitudinal study of metabolic syndrome in patients with bipolar disorder and schizophrenia. J Affect Disord 2013;150:653-8.

7. Suttajit S, Pilakanta S. Prevalence of metabolic syndrome and its association with depression in patients with schizophrenia. Neuropsychiatr Dis Treat 2013;9:941-6.

8. Morgan VA, McGrath JJ, Jablensky A, et al. Psychosis prevalence and physical, metabolic and cognitive co-morbidity: data from the second Australian national survey of psychosis. Psychol Med 2014;44:2163-76.

9. National Institute for Health and Clinical Excellence. Managing overweight and obesity in adults_lifestyle weight management services. NICE Public Health Guidance 53, 2014.

10. Newcomer JW, Weiden PJ, Buchanan RW. Switching antipsychotic medications to reduce adverse event burden in schizophrenia: establishing evidence-based practice. J Clin Psychiatry 2013;74:1108-20.

11. Fan X, Borba CP, Copeland $\mathrm{P}$, et al. Metabolic effects of adjunctive aripiprazole in clozapine-treated patients with schizophrenia. Acta Psychiatr Scand 2013;127:217-26.

12. Wang LJ, Ree SC, Huang YS, et al. Adjunctive effects of aripiprazole on metabolic profiles: comparison of patients treated with olanzapine to patients treated with other atypical antipsychotic drugs. Prog Neuropsychopharmacol Biol Psychiatry 2013;40:260-6.

13. Mizuno $Y$, Suzuki1 T, Nakagawa A, et al. Pharmacological strategies to counteract antipsychotic-induced weight gain and metabolic adverse effects in schizophrenia: a systematic review and meta-analysis. Schizophr Bull 2014;4:1385-403.

14. Barnes TRE, Paton C, Cavanagh $\mathrm{M}$, et al. A UK audit of screening for the metabolic side effects of antipsychotics in community patients. Schizophr Bull 2007;33:1397-403.

15. Mitchell AJ, Delaffon V, Vancampfort D, et al. Guideline concordant monitoring of metabolic risk in people treated with antipsychotic medication: systematic review and meta-analysis of screening practices. Psychol Med 2012;42:125-47.

16. Barnes TRE, Paton C. Improving prescribing practice in psychiatry: the experience of the Prescribing Observatory for Mental Health (POMH-UK). Int Rev Psychiatry 2011;23:328-35.

17. Joint Formulary Committee. British National Formulary (edition 68) London: BMJ Group and Pharmaceutical Press, 2014.

18. National Institute for Health and Clinical Excellence. Psychosis and schizophrenia in adults: treatment and management. NICE Clinical Guideline 178, 2014.

19. Barnes TRE, Paton C, Hancock E, et al. UK Prescribing Observatory for Mental Health. Screening for the metabolic syndrome in community psychiatric patients prescribed antipsychotics: a quality improvement programme. Acta Psychiatr Scand 2008;118:26-33.

20. Gumber R, Abbas M, Minajagi M. Monitoring the metabolic side-effects of atypical antipsychotics. Psychiatr Bull 2010;34:390-5.

21. SEPT quality account 2010-11. South Essex Partnership University NHS Foundation Trust, 2011.

22. Reddy HT, Edwards R. Screening of metabolic side effects of antipsychotics in patients with mental disorder (abstract 4P03-105). Eur Psychiatry 2011;26(Supplement 1):1274.

23. Clinical Audit Project Abstract MH 44612 13. POMH-UK Audit Topic 2f: screening for metabolic side effects of antipsychotic drugs. South Staffordshire \& Shropshire Healthcare NHS Foundation Trust, 2012.

24. Najim H. Monitoring cardiometabolic risk in schizophrenia. $\mathrm{Br} \mathrm{J}$ Psychiatry 2012;201:75-6.

25. Quality Report 2012-2013. Humber NHS Foundation Trust, 2013.21

26. Oxleas NHS. Quality Report 2012-2013, Improving Lives. Oxleas NHS Foundation Trust, 2013

27. National Institute for Health and Clinical Excellence. Schizophrenia: core interventions in the treatment and management of schizophrenia in adults in primary and secondary care. NICE Clinical Guideline 1, 2002.

28. National Institute for Health and Clinical Excellence. Schizophrenia: core interventions in the treatment and management of schizophrenia in adults in primary and secondary care. NICE Clinica Guideline 82, 2009.

29. Taylor D, Paton C, Kerwin R. The Maudsley Prescribing Guidelines, 8th edition. London and New York: Taylor \& Francis, 2005.

30. National Institute for Health and Clinical Excellence. Lipid modification: cardiovascular risk assessment and the modification of blood lipids for the primary and secondary prevention of cardiovascular disease. NICE Clinical Guideline 67, 2008

31. Royal College of Psychiatrists. Physical health in mental health: final report of a scoping group. Occasional Paper OP67. January 2009.

32. Kristensen SR, McDonald R, Sutton M. Should pay-for-performance schemes be locally designed? Evidence from the Commissioning for Quality and Innovation (CQUIN) framework. J Health Serv Res Policy 2013;18(Suppl. 2):38-49.

33. Lester $\mathrm{H}$, Campbell S. Developing quality and outcome framework (QOF) indicators and the concept of 'QOF ability'. Qual Prim Care 2010;18:103-9. 